This is the post-print version of the following article: Mora-Sanz, V; Saa, L; Briz, N; Moller, M; Pavlov, V Antibody-Directed Synthesis of Catalytic Nanoclusters for Bioanalytical Assays

ACS Applied Materials and Interfaces, 2020, 12 - 26 (28993-28999)

DOI: 10.1021/acsami.0c05229

This article may be used for non-commercial purposes in accordance with ACS Terms and Conditions for SelfArchiving. 


\section{Antibody-directed Synthesis of Catalytic Nanoclusters for Bioanalytical Assays}

Verónica Mora-Sanz ${ }^{a, b}$, Laura Saa ${ }^{a}$, Nerea Briz ${ }^{b}$, Marco Möller ${ }^{a}$ and Valeri Pavlov ${ }^{*} *$

Center for Cooperative Research in Biomaterials (CIC biomaGUNE), Basque Research and Technology Alliance (BRTA), Paseo de Miramon 182, 20014, Donostia San Sebastián, Spain

`Tecnalia, Basque Research and Technology Alliance (BRTA), Parque Tecnológico de San Sebastián, Paseo Mikeletegi 2, Donostia-San Sebastian 20009, Spain

\section{KEYWORDS}

nanocluster, antibody , photocatalytic activity, peroxidase-like activity , immunoassay 


\begin{abstract}
Synthesis of atomic nanoclusters (NCs) using proteins as scaffold has attracted great attention. Usually the synthetic conditions for the synthesis of NCs stabilized with proteins require extreme pHs or temperature. These harsh reaction conditions cause the denaturation of the proteins and end up in the loss of their biological functions. Until now there are no examples of the use of antibodies as NCs stabilisers. In this work we present the first method for the synthesis of catalytic NCs that uses antibodies for the stabilization of NCs. Anti-BSA IgG was used as a model in order to demonstrate that it is possible to use an antibody as scaffold for the synthesis of semiconductor and metallic NCs with catalytic properties. The synthesis of antibodies modified with NCs is carried out under non denaturing conditions, which do not affect the antibody structure. The resulting antibodies still maintain the affinity for target antigens and protein G. The catalytic properties of the anti-BSA IgG modified with NCs can be used to the quantification of BSA in a direct sandwich ELISA.
\end{abstract}




\section{INTRODUCTION}

Metallic and semiconductor NCs are composed of several atoms with a size smaller than 2-3 $\mathrm{nm}^{1}$ and demonstrate very peculiar optical, electronic and catalytic properties. Due to their unique properties, NCs find important applications in biodetection, bioimaging, electronics and photovoltaic ${ }^{2}$. Their specific properties arise from their subnanometric dimensions, comparable to the Fermi wavelength of electrons. The spatial isolation of free electrons in NCs provide electronic transitions tunable with size ${ }^{3}$. Several biopolymers like $\mathrm{BSA}^{45,6}$, lysozyme ${ }^{7.8}, \mathrm{GOx}^{9}, \mathrm{HRP}^{10}$, glutathione ${ }^{11}$ or DNA ${ }^{12}$ have been used as stabilizer for semiconductor and metallic clusters of atoms. The harsh conditions employed during the synthesis of semiconductor and metallic NCs, such high temperature or extremely basic $\mathrm{pH}$, with these biopolymers as scaffolds cause partial or complete loss of their initial biological properties due to denaturation. To the best of our knowledge, antibodies, which play the important part in immune response of living organisms, have never been employed as scaffolds for the synthesis of catalytic semiconductor or metallic NCs.

In the present work we used IgG as scaffold for the synthesis of NCs composed of different materials without changing its conformation and maintaining the binding for its antigen by using mild conditions during the synthesis. As model antibody-antigen system BSA and corresponding antibody (anti-BSA IgG) were selected. This system is commonly used as a model in research ${ }^{13,1,15}$. Albumin functions as a carrier protein for steroids, fatty acids, and thyroid hormones in the blood and plays a major role in stabilizing extracellular fluid volume by contributing to colloid osmotic pressure of plasma. BSA is the most abundant protein in plasma and has very important applications in cell culture, clinical diagnosis, electrophoresis chromatography and immune biochemistry ${ }^{16}$ and it is usually used as a standard protein. It is composed by a single peptide chain protein composed of 582 amino acids and its sequence is similar to that of human serum albumin (HSA) ${ }^{17}$. Two different materials were chosen for the synthesis of NCs, semiconductor NCs composed by cadmium sulfide $(\mathrm{CdS})$ and bimetallic silver/platinum $(\mathrm{Ag} / \mathrm{Pt}) \mathrm{NCs}$. 
We have chosen CdS NCs for the synthesis of CdS NCs-IgG due to their photocatalytic properties towards oxidation of $\mathrm{N}$-acetyl-3,7-dihydroxyphenoxazine (Amplex Red). CdS nanomaterials, as quantum dots (QDs) are capable of generating reactive oxygen species (ROS), as hydrogen peroxide $\left(\mathrm{H}_{2} \mathrm{O}_{2}\right)$ triggered by the exposure to ultraviolet light (UV-light) $)^{18}$. Photocatalytic activity of proteins conjugated with CdS nanoparticles (NPs) has been demonstrated with the enzyme cytochrome P450 monooxygenase ${ }^{1220}$, cytochrome c peroxidase and $\mathrm{HRP}^{21}$. Specifically conjugated with HRP, CdS nanomaterials can oxidize several typical peroxidase substrates, such as Amplex Red, guaiacol, or 2,2'-azinobis (3-ethylbenzthiazolin6-sulfonate) (ABTS) ${ }^{22}$. NCs composed by Ag and Pt have been chosen for the synthesis of Ag/Pt NCs-IgG due to their capacity to catalyze the oxidation of 3,3',5,5'-tetramethylbenzidine (TMB) in the presence of $\mathrm{H}_{2} \mathrm{O}_{2}$. Previously, $\mathrm{Ag} / \mathrm{Pt}$ bimetallic NCs, synthesized using a single stranded DNA as a template, demonstrated catalytic activity. This feature was used for the sensing of different analytes, for example mercury ions $^{23}$, L-cysteine ${ }^{24}$ or thrombin ${ }^{25}$. Herein, we use the photocatalytic and catalytic properties of semiconductor and metallic NCs embedded in antibodies in an immunoassay for the detection of target antigen. The mode that the signal is generated is similar to that of HRP-based ELISA endowing our method a promising approach for quantitative detection.

\section{RESULTS AND DISCUSSION}

Synthesis and characterization. Two different methods have been developed for the modification of AntiBSA IgG (developed in rabbit) with NCs. For the synthesis of CdS NCs-IgG (Figure 1A), a solution of $\mathrm{CdNO}_{3}(5 \mu \mathrm{L}, 0.1 \mathrm{M})$ was added to an Anti-BSA antibody solution $(200 \mu \mathrm{L}, 5 \mathrm{mg} / \mathrm{mL})$. The mixture was stirred for 15 minutes at room temperature. Then, $\mathrm{Na}_{2} \mathrm{~S}(5 \mu \mathrm{L}, 0.05 \mathrm{M})$ was added dropwise, immediately fluorescent NCs appeared. For the synthesis of Ag/Pt NCs-IgG (Figure 1B), first $\mathrm{AgNO}_{3}(50 \mu \mathrm{L}, 150 \mu \mathrm{M})$ and $\mathrm{K}_{2} \mathrm{PtCl}_{4}(120 \mu \mathrm{L}, 125 \mu \mathrm{M})$ were added to an anti-BSA antibody solution $(100 \mu \mathrm{L}$ of $1 \mathrm{mg} / \mathrm{mL})$ in 
phosphate buffer (10 mM, pH 7.0). The mixture was incubated for 30 minutes in the dark. Then, a freshly prepared solution of $\mathrm{NaBH}_{4}(30 \mu \mathrm{L}, 5 \mathrm{mM})$ was added under vigorous stirring for initiating the reduction of the metal ions, until the colour of the mixture changed from colourless to pale brown. The mixture was allowed to react for three hours at room temperature. After the reaction time both reaction mixtures were filtrated (15 minutes, $10000 \mathrm{x} \mathrm{g}$ ) using a $0.5 \mathrm{~mL}$ Amicon with a molecular weight cut-off of $30 \mathrm{kDa}$. The filtration was performed to separate the free ions from the atoms forming NCs. Control synthesis of both methods were carried out using BSA as scaffold instead anti-BSA antibody, CdS NCs-BSA and Ag/Pt NCs-BSA were produced. Fluorescent properties of CdS NCs-IgG were evaluated after filtration. The fluorescence emission could be easily seen by the naked eye under UV light and recorded by digital camera (Figure S1A). In Figure S1B the fluorescence emission spectrum of CdS NCs-IgG is shown. The maximum emission peak was observed at $610 \mathrm{~nm}$ using an excitation wavelength of $315 \mathrm{~nm}$. No fluorescence emission was observed for Ag/Pt NCs-IgG. The absorption spectra of both NCs are shown in Figure S2. 

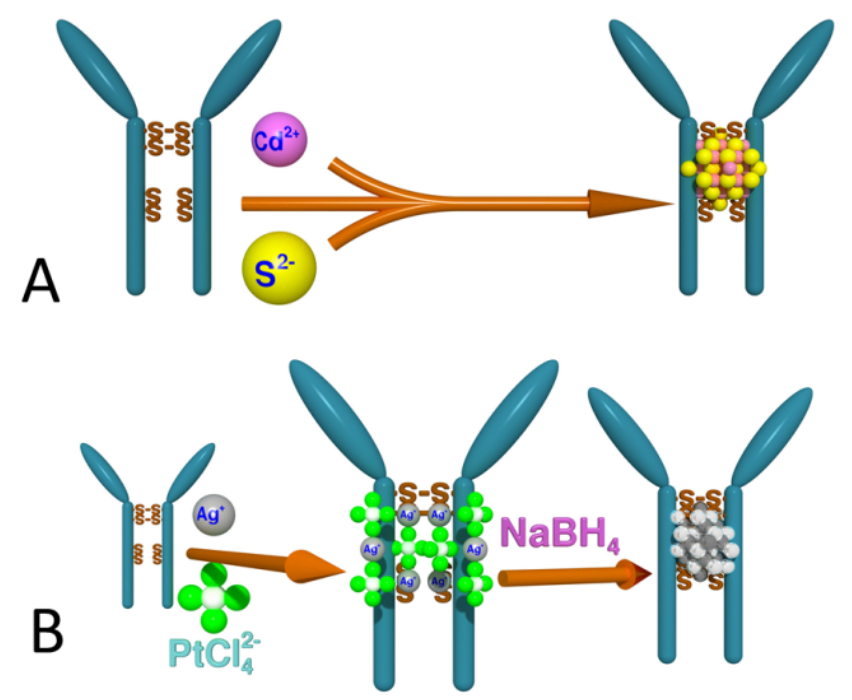

Figure 1. Scheme of the synthesis of semiconductor NCs composed by CdS (A) and bimetallic NCs composed by Ag and Pt (B). 
Characteristic absorption band for proteins appeared at $280 \mathrm{~nm}$ in both NCs, also an absoption band appeared at $425 \mathrm{~nm}$ for CdS NCs-IgG. The prepared NCs were characterized by Cryo-TEM, where the size and the morphology of the $\mathrm{CdS}$ and $\mathrm{Ag} / \mathrm{Pt}$ cores were shown clearly. The images revealed that both types of NCs have a spherical morphology (Figure 2A, Figure 2B). Figure S3 shows additional representative images of $\mathrm{Ag} / \mathrm{Pt} \mathrm{NCs-IgG} \mathrm{taken} \mathrm{in} \mathrm{other} \mathrm{areas} \mathrm{of} \mathrm{the} \mathrm{grid.} \mathrm{Based} \mathrm{on} \mathrm{the} \mathrm{statistics} \mathrm{over} 100$ individual particles, the diameter of the CdS NCs-IgG was $1.74 \pm 0.30 \mathrm{~nm}$ (Figure S4A). They have similar diameter as $\mathrm{Ag} / \mathrm{Pt} \mathrm{NCs}-\mathrm{IgG} 1.83 \pm 0.30 \mathrm{~nm}$ (Figure S4B). XPS measurements were performed to study the valence state of the metal cores of NCs-IgG (Figure 2C). The Pt4f spectra exhibit a doublet containing the Pt4f energy band at $71.1 \mathrm{eV}$ and the $\mathrm{Pt}_{4} \mathrm{f}_{5_{2}}$ at $74.3 \mathrm{eV}$ which indicates the presence of metallic state $\mathrm{Pt}^{26}$. In the $\mathrm{Pt}$ $4 \mathrm{f}_{72}$ spectrum, a broad peak was found. It was fitted by an asymmetric Lorentzian curves in the case of $\operatorname{Pt}(0)$ $(70.1 \mathrm{eV})$ and $\mathrm{Pt}(\mathrm{II})$ with a symmetric Gaussian-Lorentzian curve (71.7). This suggest that both $\mathrm{Pt}(0)$ and $\mathrm{Pt}(\mathrm{II})$ coexist as has been previously reported in bimetallic $\mathrm{NCs}^{27}$. The ratio between $\mathrm{Pt}(0) / \mathrm{Pt}$ (II) was 1.1 . The peaks of $\mathrm{Pt} 4 \mathrm{f}_{7 / 2}$ are usually found at $71 \mathrm{eV}$ and $72.4 \mathrm{eV}$ respectively. In this case they are slightly shifted to lower binding energies. These results suggests electron transfer from $\mathrm{Ag}$ to $\mathrm{Pt}$, which is related to the perturbed electronic interaction between Pt and Ag atomic orbits and forms an alloy ${ }^{28}$. The XPS spectrum of $\mathrm{Ag}$ showed a binding energy value at $368.3 \mathrm{eV}$ for $\mathrm{Ag} 3 \mathrm{~d}_{5_{2}}$ and 374.4 for $\mathrm{Ag} 3 \mathrm{~d}_{32}$ confirming the presence of elemental $\operatorname{Ag}(0)$, which agree with the results obtained with $\mathrm{AgNCs}^{29}$. The $\mathrm{Ag} 3 \mathrm{~d}_{5 / 2}$ centered at 368.3 is between the value for $\mathrm{Ag}(0)(368 \mathrm{eV})$ and $\mathrm{Ag}(\mathrm{I})(368.4 \mathrm{eV})^{30}$ which indicates the presence of $\mathrm{Ag}(\mathrm{I})$ as has been previously reported ${ }^{31}$. Cd $3 \mathrm{~d}$ peaks were fitted by two components. One associated to $\mathrm{CdS}$ bond and another one associated to $\mathrm{Cd}$ oxide or carbonate. The $\mathrm{Cd} 3 \mathrm{~d}_{3 / 2}$ energy band related to the presence of CdS bond usually appears at $411.8 \mathrm{eV}^{32}$ and for $\mathrm{CdO}$ bond at $411 \mathrm{eV}^{33}$ which agree with the experimental results. 

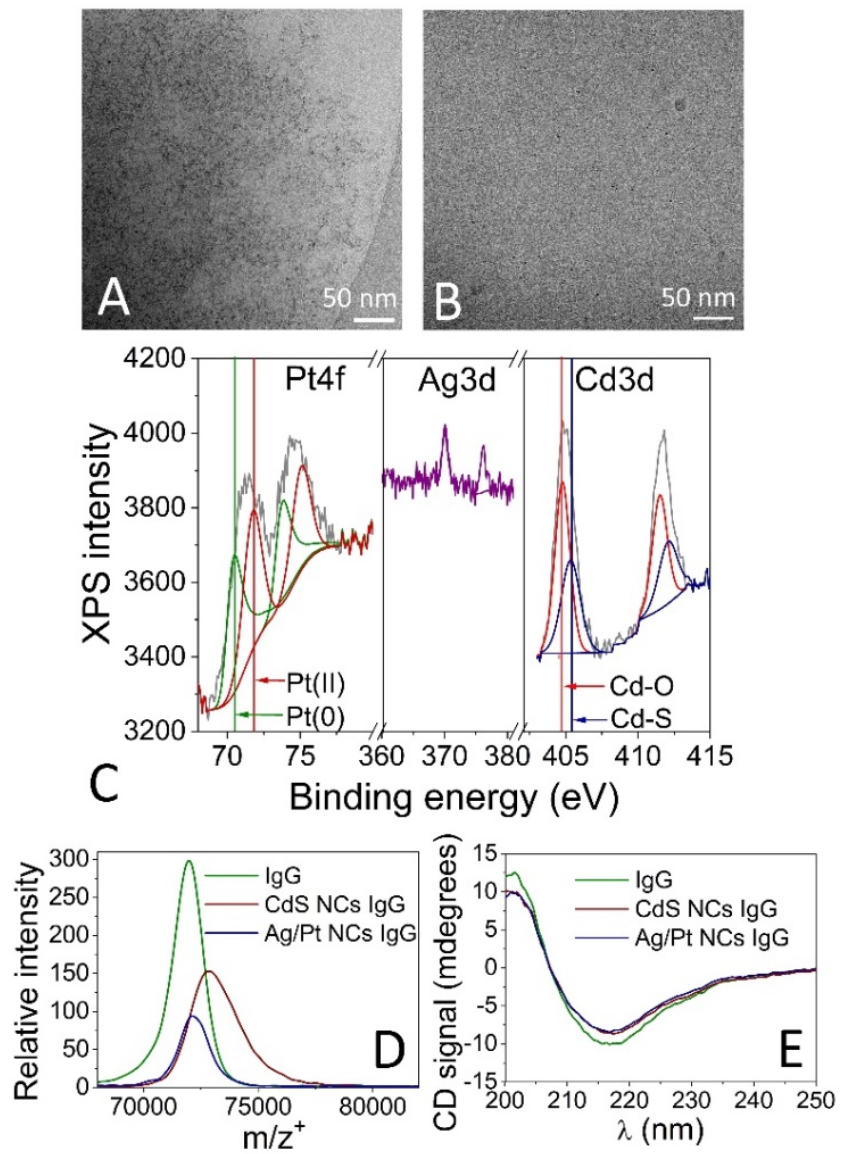

Figure 2. A. Representative TEM image of CdS NCs-IgG. B. Representative TEM image of Ag/Pt NCsIgG. C. XPS spectra of Ag/Pt NCs-IgG (left and central panel) and CdS NCs-IgG (right panel). D. MALDITOF mass spectra of Anti-BSA IgG (green), CdS NCs IgG (red) and Ag/Pt NCs IgG (blue), (m/z+, z=2). E. Far-UV CD spectra of IgG, CdS NCs-IgG and Ag/Pt NCs-IgG. 
MALDI-TOF measurements were carried out in order to make sure that the metallic nanoclusters are bound to the IgG. In Figure 2D mass spectra $\left(\mathrm{m} / \mathrm{z}^{+}, \mathrm{z}=2\right)$ for Anti-BSA IgG, CdS NCs-IgG and Ag/Pt NCs-IgG are shown. A shift in the spectra towards higher masses appeared in both NCs, in comparison with the spectrum of the unmodified Anti-BSA IgG. This result indicates that the metallic and semiconductor NCs are coordinated to the antibody. Broad spectra appeared in the three cases, being more evident in the case of NCs. The widening could be explained by two factors. On the one hand, the use of a polyclonal antibody in the synthesis with polydispersed mass. On the other hand, due to the size polydispersity of the CdS and $\mathrm{Ag} / \mathrm{Pt}$ cores observed in TEM images (Figure 2A and Figure 2B). Due to this drawback, it is not possible to give an accurate mass of the metallic core of the NCs. Nevertheless, a ratio Ag:Pt of approximately 9:1 has been calculated based on the ICP MS analysis of Ag/Pt NCs-IgG samples (Table S1). TEM images showed larger metal cores in comparison with experimental data obtained by MALDI-TOF. The ionisation of larger NCs is difficult to achieve and these ones are not detected by MALDI-TOF ${ }^{\text {sass }}$. Also, it has been described that NCs can aggregate on the carbon grids used in $\mathrm{TEM}^{136}$.

The maintenance of the conformation of the antibody is the key factor for future applications in immunoassays of the antibodies carrying NCs. CD spectroscopy can be used for characterization of the secondary structure of a protein and also to detect changes in its conformation ${ }^{37}$. Far-UV CD spectra (180$250 \mathrm{~nm}$ ) are related with the secondary structure of proteins. In the case of IgG the predominant secondary structure for IgG are the antiparallel $\beta$-sheet and random coil conformations ${ }^{38}$. A broad minimum at $218 \mathrm{~nm}$ in the far-UV CD spectra is indicative of a significant presence of $\beta$-sheets ${ }^{37}$. The introduction of metallic NCs in the structure of the anti-BSA IgG could change the conformation of the IgG and the affinity for its antigen could be reduced or completely disappear. But, CD spectra of the Anti-BSA IgG, CdS NCs-IgG and Ag/Pt NCs-IgG (Figure 2E) show no change in spectra for CdS NCs-IgG and Ag/Pt NCs-IgG, 
therefore the introduction of metallic and semiconductor NCs into the IgG structure does not cause any significant alteration in the IgG conformation.

Photocatalytic activity and catalytic activity of CdS NCs IgG and Ag/Pt NCs IgG. CdS NCs-IgG showed photocatalytic activity towards the fluorogenic substrate Amplex Red. When NCs composed by semiconductor materials such as $\mathrm{CdS}, \mathrm{CdSe}, \mathrm{ZnS}$ or $\mathrm{TiO}_{2}$ are excited with light energy greater than their band gap, electrons are promoted from the valence band to the conduction band and the generated electronhole pair can emit a photon or undergo electron transfer with the environment. Due to the latter these materials exhibit photocatalytic activity ${ }^{39}$ CdS NCs-IgG were able to oxidise Amplex Red to resofurin under UV-light (Figure 3A). 

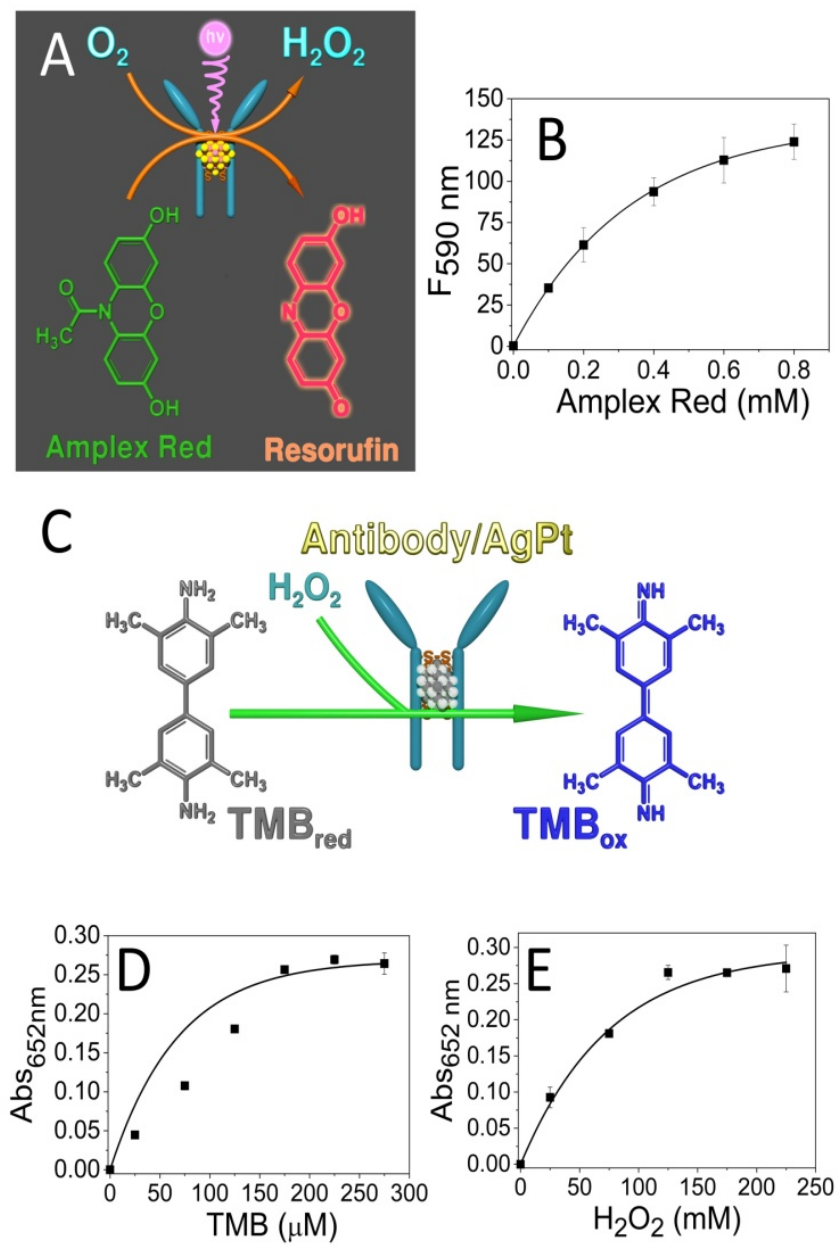

Figure 3. A. Scheme of Amplex Red oxidation to Resofurin by the photocatalytic activity of CdS NCs-IgG under UV-light. B. Scheme of TMB oxidation by the catalytic activity of Ag/Pt NCs-IgG in presence of $\mathrm{H}_{2} \mathrm{O}_{2}$. C. Fluorescence intensity obtained using different concentration of Amplex Red $\left(\lambda_{\text {exciation }}=530 \mathrm{~nm}\right.$, $\lambda$ emission=590 nm). D. Intensity of absorbance peak $(652 \mathrm{~nm})$ at different TMB concentrations and $\mathrm{E} . \mathrm{H}_{2} \mathrm{O}_{2}$ concentrations $(\lambda \mathrm{abs}=652 \mathrm{~nm})$. 
The substrate changes from colorless and non-fluorescent to pink and fluorescent $\left(\lambda_{\mathrm{ex}}=530 \mathrm{~nm}, \lambda_{\mathrm{em}}=590\right.$ $\mathrm{nm}$ ). The colour change could be easily seen by the naked eye and recorded by digital camera (Figure S5A). The photocatalytic activity of CdS NCs-IgG is dependent of Amplex Red concentration. This dependence was measured while varying the Amplex Red concentration from 0 to $0.8 \mathrm{mM}$. The CdS NCsIgG concentration was kept constant to $500 \mu \mathrm{g} / \mathrm{mL}$ (referred to Anti-BSA IgG concentration). The solution containing CdS NCs-IgG and Amplex Red was irradiated with UV-light $\left(\lambda_{\mathrm{ex}}=365 \mathrm{~nm}\right)$ for 20 minutes and the fluorescent spectra were recorded (Figure S5B). The system follows a Michaelis-Menten kinetics, the reaction rate (fluorescence of Amplex Red) increases with increasing substrate concentration (Figure 3B). The stability of CdS NCs-IgG under UV light was studied, by measuring both the fluorescence and the photocatalytic activity of CdS NCs-IgG after incubation of the samples under UV light for different times. We observed that while fluorescence is decreasing with irradiation time, the photocatalytic activity of NCs remains stable and is not affected by a previous UV light irradiation (Figure S6).

It was found that $\mathrm{Ag} / \mathrm{Pt} \mathrm{NCs}$ IgG had enzymatic peroxidase activity, like the commonly used enzyme HRP. These NCs catalysed the oxidation of the chromogenic substrate TMB with $\mathrm{H}_{2} \mathrm{O}_{2}$ which changes its colour from colourless to blue $\left(\lambda_{\mathrm{ats}}=652 \mathrm{~nm}\right)$ (Figure $\left.3 \mathrm{C}\right)$. The colour change could be easily seen by the naked eye and recorded by digital camera (Figure S7). The peroxidase-like activity of Ag/Pt NCs-IgG is dependent on TMB and $\mathrm{H}_{2} \mathrm{O}_{2}$ concentration. On the one hand, the TMB concentration dependence was measured by maintaining the $\mathrm{H}_{2} \mathrm{O}_{2}$ amount fixed at $125 \mathrm{mM}$ and by changing the TMB concentration from 0 to $275 \mu \mathrm{M}$. On the other hand, the $\mathrm{H}_{2} \mathrm{O}_{2}$ concentration dependence was measured by maintaining the TMB concentration fixed at $200 \mathrm{mM}$ and by varying the $\mathrm{H}_{2} \mathrm{O}_{2}$ concentration from 0 to $225 \mathrm{mM}$. In both cases the $\mathrm{Ag} / \mathrm{Pt} \mathrm{NCs}$ IgG concentration was kept constant to $33 \mu \mathrm{g} / \mathrm{mL}$ (referred to Anti-BSA IgG concentration). Michaelis-Menten like curves for both substrates were observed (Figure 3D and Figure 3E). With the Lineweaver-Burk equation the Michaelis-Menten constant $\left(K_{m}\right)$ was obtained. $K_{m}$ is an indicator of enzyme affinity to substrates. Lower the $K_{m}$ stronger affinity and vice versa ${ }^{40,41}$. In Table S2 the $K_{m}$ values 
for $\mathrm{Ag} / \mathrm{Pt} \mathrm{NCs}-\mathrm{IgG}$ and $\mathrm{HRP}^{42}$ are shown. The $K_{m}$ value of $\mathrm{Ag} / \mathrm{Pt} \mathrm{NCs}$ IgG was almost the same as that of HRP towards TMB, which indicates that $\mathrm{Ag} / \mathrm{Pt} \mathrm{NCs}-\mathrm{IgG}$ had similar affinity as HRP to TMB as a substrate, while the $K_{m}$ for $\mathrm{Ag} / \mathrm{Pt} \mathrm{NCs} \mathrm{IgG}$ was significantly higher than that for $\mathrm{HRP}$ towards $\mathrm{H}_{2} \mathrm{O}_{2}$, indicating that $\mathrm{Ag} / \mathrm{Pt} \mathrm{NCs} \mathrm{IgG}$ had a lower affinity for $\mathrm{H}_{2} \mathrm{O}_{2}$ than $\mathrm{HRP}$. This phenomena has been also observed in others nanozymes ${ }^{43,44}$.

Affinity of IgG modified with NCs to Protein G. The affinity of the $\mathrm{F}_{\mathrm{c}}$ region of IgG produced in rabbit for Protein G was also used to check if the introduction of NCs inside of the antibody causes any damage to the structure of the $F_{c}$ region. The $F_{c}$ region of an $\mathrm{IgG}$ binds specifically to protein G. Polyvinyl chloride microbeads decorated with Protein G were incubated with the antibodies carrying semiconductor and bimetallic NCs as described in the experimental section. NCs of $\mathrm{CdS}$ and $\mathrm{Ag} / \mathrm{Pt}$ produced using BSA as scaffold were synthesized in order to be used as controls. These BSA-stabilized NCs were characterized by TEM and XPS, to ensure that they have similar properties to those stabilized in IgG. (Fig S8 and S9). BSA does not have any affinity for Protein G, therefore any binding to the protein $\mathrm{G}$ should be detected. The CdS NCs stabilized using BSA are fluorescent ${ }^{6}$ and have catalytic activity towards the oxidation of Amplex Red in the presence of $\mathrm{H}_{2} \mathrm{O}_{2}$ (Fig S10). BSA carrying $\mathrm{Ag} / \mathrm{Pt}$ NCs synthesized by us showed catalytic properties with respect to oxidation of TMB in presence of $\mathrm{H}_{2} \mathrm{O}_{2}$. (Fig S11) The fluorescent properties of the CdS NCs-IgG and CdS NCs-BSA and the catalytic properties of Ag/Pt NCs-IgG and Ag/Pt NCs-BSA were used to verify that the IgG modified with NCs bind specifically to Protein G microbeads while BSA carrying $\mathrm{CdS}$ and $\mathrm{Ag} / \mathrm{Pt}$ do not adsorb non-specifically on protein $\mathrm{G}$ microbeads.

The fluorescence spectra of the CdS NCs-IgG and CdS NCs-BSA incubated with the microbeads were measured using an excitation wavelength of $315 \mathrm{~nm}$. Also, the fluorescence of CdS NCs-IgG and CdS NCs-BSA in solution without microbeads was measured. The fluorescent spectra are showed in Figure 4A. The spectrum of CdS NCs-IgG in solution (blue line) is practically the same as the spectrum of Protein G microbeads after washing which were pre-incubated with CdS NCs-IgG (grey line). When BSA carrying 
CdS NCs was incubated with Protein G microbeads, practically no fluorescence was observed after washing (red line). Taking into consideration that BSA carrying CdS NCs show good fluorescence in solution (green line) we conclude that fluorescence shown by Protein G microbeads modified with $\mathrm{CdS}$ NCs-IgG is attributed to specific binding of CdS NCs-IgG to protein $G$ and $F_{c}$ region of Anti-BSA IgG modified with CdS NCs was not altered.

The catalytic activity with TMB in the presence of $\mathrm{H}_{2} \mathrm{O}_{2}$ was used to probe the binding of $\mathrm{Ag} / \mathrm{Pt} \mathrm{NCs}-\mathrm{IgG}$ and $\mathrm{Ag} / \mathrm{Pt} \mathrm{NCs}-\mathrm{BSA}$ to Protein $\mathrm{G}$ microbeads. The microbeads were incubated with $\mathrm{Ag} / \mathrm{Pt} \mathrm{NCs}-\mathrm{IgG}$ and Ag/Pt NCs-BSA. The supernatant was discarded and microbeads were washed by centrifuging three times. Next, $100 \mu \mathrm{L}$ of a solution of TMB $(200 \mu \mathrm{M})$ and $\mathrm{H}_{2} \mathrm{O}_{2}(125 \mathrm{mM})$ in acetate buffer $(10 \mathrm{mM}, \mathrm{pH}=4.0)$ was added to pellets. The activity of free $\mathrm{Ag} / \mathrm{Pt} \mathrm{NCs}-\mathrm{IgG}$ and $\mathrm{Ag} / \mathrm{Pt} \mathrm{NCs}-\mathrm{BSA}$ in solution was determined by the addition of the same solution of $\mathrm{TMB}$ and $\mathrm{H}_{2} \mathrm{O}_{2}$. In Figure $4 \mathrm{~B}$ the absorbance spectra are showed. The catalytic activity of $\mathrm{Ag} / \mathrm{Pt} \mathrm{NCs}-\mathrm{IgG}$ in solution (blue line) is practically the same as that of the $\mathrm{Ag} / \mathrm{Pt} \mathrm{NCs}-$ IgG incubated with Protein G microbeads (grey line). However, with Ag/Pt NCs-BSA incubated with Protein $\mathrm{G}$ microbeads (red line) no catalytic activity was detected. Free Ag/Pt NCs-BSA in solution demonstrates catalytic activity (green line). These results indicate that the $\mathrm{F}_{\mathrm{c}}$ structure of the $\operatorname{IgG}$ was not changed during the synthesis of CdS NCs-IgG and Ag/Pt NCs-IgG because the IgG still has affinity for Protein G, moreover this binding is specific for IgG because no binding of CdS NCs-BSA and Ag/Pt NCsBSA to Protein G microbeads was observed. 

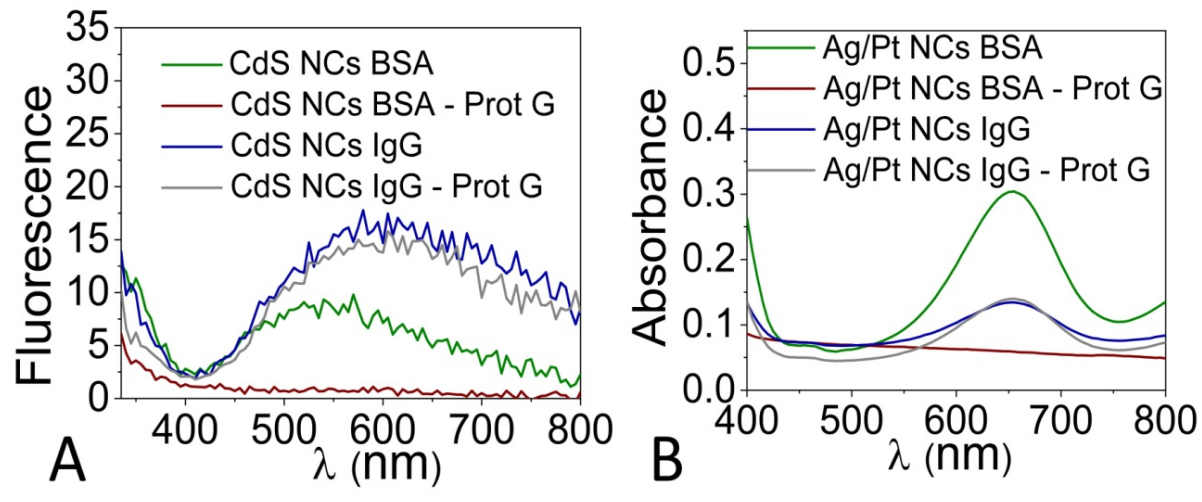

Figure 4. A. Fluorescence spectra $(\lambda e x=315 \mathrm{~nm})$ of CdS NCs-IgG and CdS-BSA incubated with microbeads decorated with Protein G and free in solution. B. Catalytic properties of Ag/Pt NCs-IgG and $\mathrm{Ag} / \mathrm{Pt}$ NCs-BSA with TMB in presence of $\mathrm{H}_{2} \mathrm{O}_{2}$ incubated with microbeads decorated with Protein $\mathrm{G}$ and free in solution. 
Interaction of Anti-BSA IgG modified with CdS and Ag/Pt NCs with their antigen. In order to know if the antibody maintains its affinity for target analyte after the introduction of NCs in its structure a direct immunoassay was performed. Figure 5A represents the method used to evaluate the binding of CdS NCsIgG with different amounts of BSA immobilized on the surface of plastic microplates obtained by incubation with varying concentrations of BSA (from 0 to $10 \mu \mathrm{g} / \mathrm{mL}$ ) in buffered solutions.

The higher was the amount of BSA adsorbed the greater photooxidation rate of Amplex Red to Resorufin was observed. The calibration curve shows (Figure 5B) that the fluorescence emission at $\lambda_{\mathrm{cm}}=590 \mathrm{~nm}$ $\left(\lambda_{\mathrm{u}}=530 \mathrm{~nm}\right)$ increases with BSA concentration. The fluorescence values were recorded after 30 minutes of incubation of aqueous solutions (365 nm) of Amplex Red, placed in microplates wells, under UV-light. In Figure 5C the scheme of the direct immunoassay based on the catalytic properties of $\mathrm{Ag} / \mathrm{Pt}$ NCs-IgG is showed. The amount of BSA adsorbed was controlled by incubation with solutions containing varying concentrations of BSA ( 0 to $1400 \mathrm{ng} / \mathrm{mL}$ ). When the surface coverage of BSA immobilized on the microplate increased, the oxidation rate of TMB increased proportionally measured by UV-Vis spectroscopy $\left(\lambda_{\mathrm{san}}=652 \mathrm{~nm}\right)$. The calibration curve shows (Figure 5D) how the initial oxidation rate of TMB (measured during 4 minutes) increased with increasing BSA concentration. In Figure S12 the dependence of TMB absorbance on time for every BSA concentration is represented. Comparing both immunoassays, the method using $\mathrm{Ag} / \mathrm{Pt}$ NCs-IgG showed several advantages. First, it is faster, because only 4 minutes of catalytic oxidation of TMB with $\mathrm{H}_{2} \mathrm{O}_{2}$ were needed to calculate the initial oxidation rate. In case of assay based on CdS NCs-IgG the photooxidation with Amplex Red required 30 minutes to produce calibration plot. 

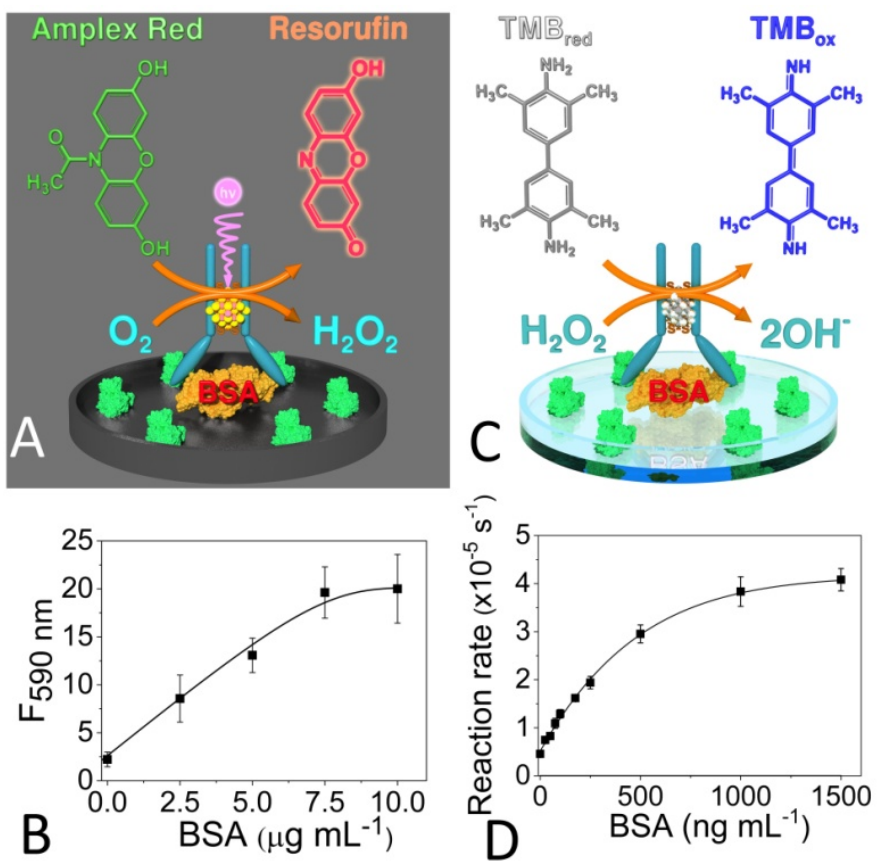

Figure 5. A. Direct immunoassay for BSA based on the photocatalytic properties of CdS NCs-IgG. B. Variation of the fluorescence of Amplex Red as a function of BSA concentration $(\lambda \mathrm{ex}=530 \mathrm{~nm}, \lambda \mathrm{em}=590$ $\mathrm{nm}$ ). At least three replicates were performed to obtain the readout signal. (C) Direct immunoassay for BSA based on the catalytic properties of Ag/Pt NCs-IgG. (D) Reaction rate of TMB oxidation as a function of BSA concentration $(\lambda \mathrm{abs}=652 \mathrm{~nm})$. At least three replicates were performed to obtain the readout signal. 
The use of the UV-light made the photocatalytic method technically more complicated. The same intensity of light must reach all wells, so the position of the microplate with respect to the UV-light source is critical for the reproducibility of the method. Due to its use greater relative standard deviations were achieved with CdS NCs-IgG. Due to better sensitivity for immobilized BSA, the lower standard deviations and the possibility to perform the assay without the source of the UV-light, $\mathrm{Ag} / \mathrm{Pt} \mathrm{NCs}-\mathrm{IgG}$ were used in further studies.

Comparison of utility in sandwich ELISA of different methods for labelling antibodies with catalytic elements. The performance of a direct sandwich ELISA carried out using as detection antibody Ag/Pt NCsIgG was compared with a system using as detection antibody an IgG labelled with HRP. First, we developed the direct sandwich ELISA assay using Ag/Pt NCs Anti-BSA IgG (from rabbit) (Figure 6A) by varying the concentration of the detection antibody in order to obtain its optimum concentration. Capture antibody from chicken was immobilised on the surface of microplate and the surface of the well is blocked with casein. Then the target analyte (0 and $200 \mathrm{ng} / \mathrm{mL}$ ) was added in different microplate wells. Later, Ag/Pt NCs Anti-BSA IgG (from rabbit) was added. TMB solution was added to the microplate and the increase of the oxidation rate of TMB was measured for 4 minutes on the standard plate reader. In order to optimise the assay varying concentrations of Ag/Pt NCs Anti-BSA IgG were added. In Figure S13A the reaction rate of TMB oxidation was represented for the assays with and without the target analyte for each Ag/Pt NCs-IgG concentration. The highest ratio between two signals observed with and without the target analyte was 5.23 (Figure S13B). 


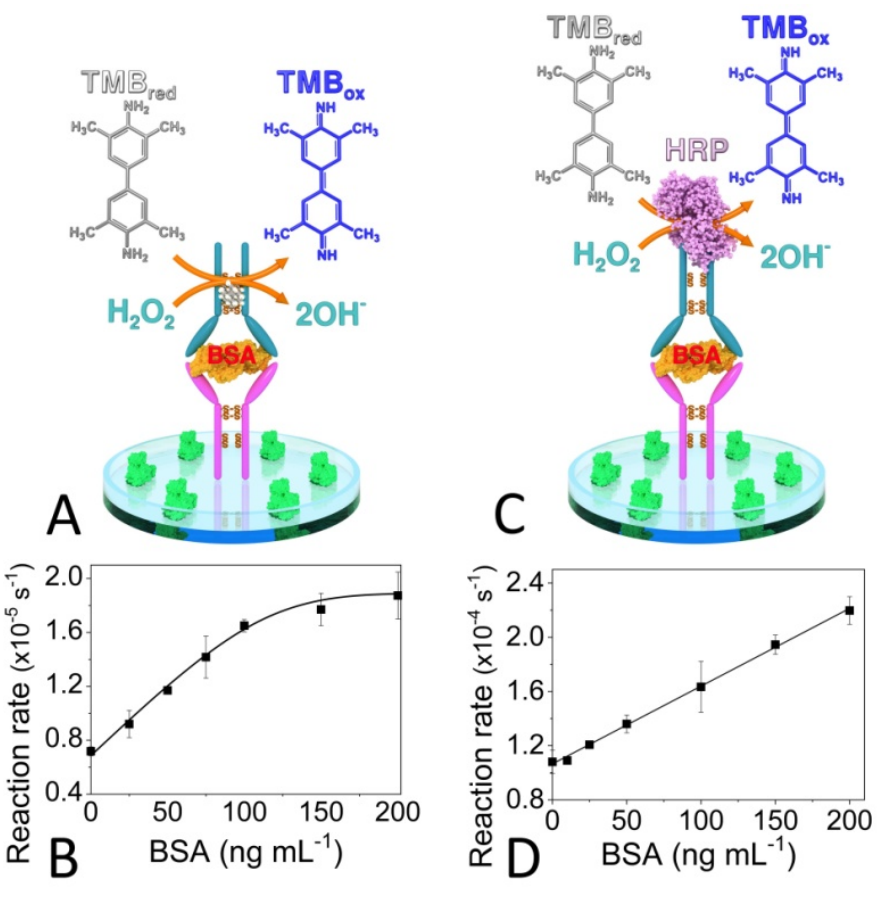

Figure 6. A. Direct sandwich ELISA for BSA detection using Ag/Pt NCs Anti-BSA IgG as the primary antibody. B. Calibration curve of the direct sandwich ELISA system based on Ag/Pt NCs Anti-BSA IgG using BSA as a target analyte. Five replicates were used to calculate value of the readout signal. C. Direct sandwich ELISA for BSA detection using antibody labelled with HRP as the primary antibody. D. Calibration curve of the direct sandwich ELISA system based on Anti-BSA IgG-HRP using BSA as a target analyte. Five replicates were used to calculate value of the readout signal. 
Having obtained the optimal concentration of Ag/Pt NCs Anti-BSA IgG we performed calibration curve of the system with different concentrations of BSA, which is shown in Figure 6B. According to the figure, the linear range was up to $100 \mathrm{ng} / \mathrm{mL}$ and the limit of detection was $10.4 \mathrm{ng} / \mathrm{mL}$. Second, we studied performance of the conventional direct sandwich ELISA (Figure 6C). Capture antibodies for BSA were passively attached to the solid phase of a microplate and the surface was blocked with casein to prevent non-specific adsorption. Next after incubation with BSA (0 and $200 \mathrm{ng} / \mathrm{mL}$ ), antibody for BSA (from rabbit) labelled with HRP, which is the standard enzymatic label employed broadly in ELISA, was incubated. Finally, TMB was added and the reaction rate was measured during 4 minutes in the standard plate reader. We compared the reaction rate of TMB oxidation demonstrated by two samples containing 0 and $200 \mathrm{ng} / \mathrm{mL}$ of BSA in order to find out the optimum concentration of primary antibody labelled with HRP in order to avoid its non-specific adsorption and obtain the highest ratio between the readout signals. In Figure S14A the reaction rate of TMB oxidation was represented for the assays with and without target analyte for each anti-BSA IgG concentration. The best ratio between two readout signals (3.55) was obtained for a primary antibody/HRP conjugated concentration of $0.033 \mu \mathrm{g} / \mathrm{mL}$ as one can see in Figure S14B. Having obtained the optimum concentration of Anti-BSA IgG-HRP we performed calibration curve of the system with different concentrations of BSA, which is shown in Figure 6D. According to the figure the limit of detection was $52.03 \mathrm{ng} / \mathrm{mL}$.

According to our results direct sandwich ELISA based on Ag/Pt NCs Anti-BSA IgG demonstrates the better performance with respect the target analyte. This assay showed higher signal background ratio. In both assays capture and primary antibodies were the same (in case of the ELISA employing Ag/Pt NCs Anti-BSA IgG antibody used for its preparation was the same as the primary antibodies in the other assay). This means that the comparison between two different assays was performed under the most similar conditions. In order to perform the direct sandwich assay based on Anti-BSA IgG/HRP conjugate the commercially available kit from Abcam specifically designed and optimised for conjugation of antibodies 
with HRP was employed to produce Anti-BSA IgG/HRP conjugate. Nevertheless the assay based on "Abcam modified" antibodies showed lower signal noise ratio than that of our assay employing Ag/Pt NCs IgG. The LOD was improved up to 5 times by using Ag/Pt NCs-IgG than the system using IgG-HRP. The synthesis of $\mathrm{Ag} / \mathrm{Pt} \mathrm{NCs}$ IgG provides a new kind of detection antibodies that increases the sensitivity of conventional ELISAs.

\section{CONCLUSIONS}

This study provides the first methods for the synthesis of semiconductor and bimetallic NCs using antibodies as scaffolds. The non-denaturing conditions used during the synthesis make that the antibody structure remains unalterable after the modification, thus antibody still has affinity for its target analyte and Protein G. The CdS NCs-IgG show photocatalytic properties and they are able to oxidize the fluorogenic substrate Amplex Red triggered by the exposition to UV-light. The Ag/Pt NCs-IgG exhibit peroxidase-like

activity and oxidize the chromogenic substrate TMB in presence of $\mathrm{H}_{2} \mathrm{O}_{2}$. Both reactions follow a Michaelis-Menten kinetics, the reaction rate increases with substrate concentration. The Ag/Pt NCs-IgG can be used as detection antibody in a direct sandwich ELISA and the concentration of the target analyte can be related with the reaction rate of TMB oxidation. In comparison with conventional methods used in sandwich ELISA for detection, such as the modification of the primary antibody with HRP, this new detection method provides better sensitivity.

\section{ASSOCIATED CONTENT}


Supporting Information. Experimental section, absorption and fluorescence spectra of CdS NCs-IgG and $\mathrm{Ag} / \mathrm{Pt}$ NCs-IgG, ICP analysis, optimization experiments, characterization of BSA-stabilized NCs, and size distribution of NCs. The following files are available free of charge.

\section{AUTHOR INFORMATION}

\section{Corresponding Author}

*Valeri Pavlov email: vpavlov@cicbiomagune.es

\section{Author Contributions}

Pavlov, V. and Briz, N. conceived and planned the research. Mora-Sanz, V. and Saa L. performed experimental research and wrote the manuscript, Möller, M. participated in characterization of atomic clusters grown in BSA. All authors have given approval to the final version of the manuscript.

\section{Funding Sources}

Spanish Ministry of Science, Innovation and Universities (Project BIO2017-88030-R). and Maria de Maeztu Units of Excellence Program- Grant No. MDM-2017-0720.

\section{ACKNOWLEDGMENT}

The authors gratefully acknowledge support from the Spanish Ministry of Science, Innovation and Universities (Project BIO2017-88030-R). We thank Dr. Di Silvio and Dr. D. Gil for support with XPS and TEM measurements. This work was performed under the Maria de Maeztu Units of Excellence Programme - Grant No. MDM-2017-0720 Ministry of Science, Innovation and Universities.

\section{REFERENCES}


(1) Chen, L.-Y.; Wang, C.-W.; Yuan, Z.; Chang, H.-T. Fluorescent Gold Nanoclusters: Recent Advances in Sensing and Imaging. Anal. Chem. 2015, 87 (1), 216-229. https://doi.org/10.1021/ac503636j.

(2) Jariwala, D.; Sangwan, V. K.; Lauhon, L. J.; Marks, T. J.; Hersam, M. C. Carbon Nanomaterials for Electronics, Optoelectronics, Photovoltaics, and Sensing. Chem. Soc. Rev. 2013, 42 (7), 2824. https://doi.org/10.1039/c2cs35335k.

(3) Chib, R.; Butler, S.; Raut, S.; Shah, S.; Borejdo, J.; Gryczynski, Z.; Gryczynski, I. Effect of Quencher, Denaturants, Temperature and $\mathrm{pH}$ on the Fluorescent Properties of BSA Protected Gold Nanoclusters. J. Lumin. 2015, 168, 62-68. https://doi.org/10.1016/j.jlumin .2015.07.030.

(4) Xie, J.; Zheng, Y.; Ying, J. Y. Protein-Directed Synthesis of Highly Fluorescent Gold Nanoclusters. J. Am. Chem. Soc. 2009, 131 (3), 888-889. https://doi.org/10.1021/ja806804u.

(5) Das, T.; Ghosh, P.; Shanavas, M. S.; Maity, A.; Monda, S.; Purkayastha, P. Protein-Templated Gold Nanoclusters: Size Dependent Inversion of Fluorescence Emission in the Presence of Molecular Oxygen. Nanoscale 2012, 4, 6018-6024. https://doi.org/10.1039/c2nr31271a.

(6) Han, S.-Q.; Liu, J. L.; Gan, Z.-G.; Liang, J. G.; Zhao, S. M. Application of Luminescent BSACapped CdS Quantum Dots as a Fluorescence Probe for the Detection of $\mathrm{Cu} 2+$. J. Chinese Chem. Soc. 2015, 55, 1069-1073. https://doi.org/10.1002/jccs.200800156.

(7) Ghosh, R.; Sahoo, A. K.; Ghosh, S. S.; Paul, A.; Chattopadhyay, A. Blue-Emitting Copper Nanoclusters Synthesized in the Presence of Lysozyme as Candidates for Cell Labeling. ACS Appl. Mater. Interfaces 2014, 6, 3822-3828. https://doi.org/10.1021/am500040t.

(8) Zhou, T.; Huang, Y.; Cai, Z.; Luo, F.; Yang, C. J.; Chen, Y. Facile Synthesis of Red-Emitting 
Lysozyme-Stabilized Ag Nanoclusters. Nanoscale 2012, $4 \quad$ (17), 5312. https://doi.org/10.1039/c2nr31449e.

(9) Xia, X.; Long, Y.; Wang, J. Glucose Oxidase-Functionalized Fluorescent Gold Nanoclusters as Probes for Glucose. Anal. Chim. Acta 2013, 772, 81-86. https://doi.org/10.1016/j.aca.2013.02.025.

(10) Wen, F.; Dong, Y.; Feng, L.; Wang, S.; Zhang, S.; Zhang, X. Horseradish Peroxidase Functionalized Fluorescent Gold Nanoclusters for Hydrogen Peroxide Sensing Characterization. Anal. Chem. 2011, $83,1193-1196$.

(11) Ding, W.; Guan, L.; Han, J.; Mangala, R.; Luo, Z. Fluorescence Chemosensing of Water-Soluble Ag14nanoclusters for Lysozyme and Hg2+ions. Sensors Actuators B 2017, 250, 364-371. https://doi.org/10.1016/j.snb.2017.04.172.

(12) Schultz, D.; Gardner, K.; Oemrawsingh, S.R.O. Markeševic“, N.; Olsson, K.; Debord, M.; Bouwmeester, D.; Gwinn, E. Evidence for Rod-Shaped DNA-Stabilized Silver Nanocluster Emitters. Adv. Mater. 2013, 25, 2797-2803. https://doi.org/10.1002/adma.201204624.

(13) Kessler, S. W. Rapid Isolation of Antigens from Cells with A Staphylococcal Protein A-Antibody Adsorbent: Parameters of the Interaction of Antibody-Antigen Complexes with Protein A. $J$. Immunol. 1975, 115, 1617-1624.

(14) Barroso, J.; Saa, L.; Grinyte, R.; Pavlov, V. Photoelectrochemical Device Based on Modified by Osmium Polymer to Detection of Enzymatic Activities. Biosens. Bioelectron. 2016, 77, 323-329.

(15) Malashikhina, N.; Garai-Ibabe, G.; Pavlov, V. Unconventional Application of Conventional Enzymatic Substrate: First Fluorogenic Immunoassay Based on Enzymatic Formation of Quantum Dots. Anal. Chem. 2013, 85, 6866-6870. https://doi.org/10.1021/ac4011342. 
(16) Deschaume, O.; Shafran, K. L.; Perry, C. C. Interactions of Bovine Serum Albumin with Aluminum Polyoxocations and Aluminum Hydroxide. Langmuir 2006, 22 (24), 10078-10088. https://doi.org/10.1021/la061285h.

(17) Huang, D.; Geng, F.; Liu, Y.; Wang, X.; Jiao, J.; Yu, L. Biomimetic Interactions of Proteins with Functionalized Cadmium Sulfide Quantum Dots. Colloids Surfaces A Physicochem. Eng. Asp. 2011, 392 (1), 191-197. https://doi.org/10.1016/j.colsurfa.2011.09.054.

(18) Ipe, B. I.; Lehnig, M.; Niemeyer, C. M. On the Generation of Free Radical Species from Quantum Dots. Small 2005, 1 (7), 706-709. https://doi.org/10.1002/smll.200500105.

(19) Slocik, J. M.; Govorov, A. O.; Naik, R. R. Photoactivated Biotemplated Nanoparticles as an Enzyme Mimic. Angew. Chemie 2008, 47 (29), 5335-5339. https://doi.org/10.1002/anie.200800023.

(20) Gandubert, V. J.; Torres, E.; Niemeyer, C. M. Investigation of Cytochrome P450-Modified Cadmium Sulfide Quantum Dots as Photocatalysts. J. Mater. Chem. 2008, 18 (32), 3824-3830. https://doi.org/10.1039/b805526b.

(21) Fruk, L., Rajendran, V., Spengler, M. and Niemeyer, C. M. Light-Induced Triggering of Peroxidase Activity Using Quantum Dots. ChemBioChem 2007, 8 (18), 2195-2198. https://doi.org/10.1002/cbic.200700594.

(22) Fruk, L.; Rajendran, V.; Spengler, M.; Niemeyer, C. M. Light-Induced Triggering of Peroxidase Activity Using Quantum Dots. ChemBioChem 2007, 8 (18), 2195-2198. https://doi.org/10.1002/cbic.200700594.

(23) Wu, L. L.; Wang, L. Y.; Xie, Z. J.; Xue, F.; Peng, C. F. Colorimetric Detection of Hg2+based on Inhibiting the Peroxidase-like Activity of DNA-Ag/Pt Nanoclusters. $R S C A d v . \mathbf{2 0 1 6}, 6$ (79), 75384- 
75389. https://doi.org/10.1039/c6ra12597b.

(24) Wu, L. L., Wang, L. Y., Xie, Z. J., Pan, N. and Peng, C. F. Colorimetric Assay of L-Cysteine Based on Peroxidase-Mimicking DNA-Ag/Pt Nanoclusters. Sensors Actuators, B Chem. 2016, 235, 110116. https://doi.org/10.1016/j.snb.2016.05.069.

(25) Zheng, C., Zheng, A. X., Liu, B., Zhang, X. L.; He, Y., Li, J., Yang, H. H. and Chen, G. One-Pot Synthesized DNA-Templated Ag/Pt Bimetallic Nanoclusters as Peroxidase Mimics for Colorimetric Detection of Thrombin. Chem. Commun. 2014, 50 (86), 13103-13106. https://doi.org/10.1039/c4cc05339g.

(26)

Davis, D. J.; Kyriakou, G.; Lambert, R. M. Uptake of n -Hexane , 1-Butene, and Toluene by Au / Pt Bimetallic Surfaces: A Tool for Selective Sensing of Hydrocarbons under High-Vacuum Conditions. J. Phys. Chem B 2006, 110, 11958-11961.

(27) Xu, J. B.; Zhao, T. S.; Liang, Z. X. Synthesis of Active Platinum - Silver Alloy Electrocatalyst toward the Formic Acid Oxidation Reaction. J. Phys. Chem C 2008, 112, 17362-17367.

(28) Zeng, J.; Yang, J.; Lee, J. Y.; Zhou, W. Preparation of Carbon-Supported Core - Shell Au - Pt Nanoparticles for Methanol Oxidation Reaction: The Promotional Effect of the Au Core. J. Phys. Chem B 2006, 24606-24611.

(29) Yang, X.; Gan, L.; Han, L.; Wang, E.; Wang, J. High-Yield Synthesis of Silver Nanoclusters Protected by DNA Monomers and DFT Prediction of Their Photoluminescence Properties **. Angew. Chem. Int. Ed. 2013, 52, 2022-2026. https://doi.org/10.1002/anie.201205929.

(30) Volkov, I. L.; Smirnova, A.; Makarova, A. A.; Reveguk, Z. V; Ramazanov, R. R.; Usachov, D. Y.; Adamchuk, V. K.; Kononov, A. I. DNA with Ionic, Atomic, and Clustered Silver: An XPS Study. 
J. Phys. Chem B 2017, 121, 2400-2406. https://doi.org/10.1021/acs.jpcb.6b11218.

(31) Aires, A.; Llarena, I.; Moller, M.; Castro-Smirnov, J.; Cabanillas-Ginzalez, J.; Cortajarena, A. L. A Simple Approach to Design Proteins for the Sustainable Synthesis of Metal Nanoclusters. Angew. Chem. Int. Ed. 2019, 58, 6214-6219. https://doi.org/10.1002/anie.201813576.

(32) Barreca, D.; Gasparotto, A.; Tondello, E. Nanostructured Cadmium Sulfide Thin Films by XPS. Surf. Sci. Spectra 2003, 9, 46-53. https://doi.org/10.1116/11.20030116.

(33) Subramanyam, T. K.; Naidu, B. S.; Uthanna, S. Studies on Dc Magnetron Sputtered Cadmium Oxide Films. Appl. Surf. Sci. 2001, 169-170, 529-534.

(34) Wang, C.; Ling, L.; Yao, Y.; Song, Q. One-Step Synthesis of Fluorescent Smart ThermoResponsive Copper Clusters: A Potential Nanothermometer in Living Cells. Nano Res. 2015, 8, 1975-1986. https://doi.org/10.1007/s12274-015-0707-0.

(35) Jia, X.; Li, J.; Wang, E. Cu Nanoclusters with Aggregation Induced Emission Enhancement. Small 2013, 9, 3873-3879. https://doi.org/10.1002/smll.201300896.

(36) Lin, Y.; Chen, P.; Yuan, Z.; Ma, J.; Chang, H. The Isomeric Effect of Mercaptobenzoic Acids on the Preparation and Fluorescence Properties of Copper Nanoclusters. Chem. Commun. 2015, 51, $11983-$ 11986. https://doi.org/10.1039/C5CC02342D.

(37) Joshi, V.; Shivach, T.; Yadav, N.; Rathore, A. S. Circular Dichroism Spectroscopy as a Tool for Monitoring Aggregation in Monoclonal Antibody Therapeutics. Anal. Chem. 2014, 86 (23), 1160611613.

(38) Vermeer, A. W. P.; Bremer, M. G. E. G.; Norde, W. Structural Changes of IgG Induced by Heat Treatment and by Adsorption onto a Hydrophobic Teflon Surface Studied by Circular Dichroism 
Spectroscopy. Biochim. Biophys. Acta 1998, 1425, 1-12.

(39) Rajendran, V.; Konig, A.; Rabe, K. S.; Niemeyer, C. M. Photocatalytic Activity of ProteinConjugated CdS Nanoparticles. Small 2010, $6 \quad$ (18), 2035-2040. https://doi.org/10.1002/smll.201000690.

(40) Jiang, X.; Sun, C.; Guo, Y.; Nie, G.; Xu, L. Peroxidase-like Activity of Apoferritin Paired Gold Clusters for Glucose Detection. Biosens. Bioelectron. 2015, 64, 165-170. https://doi.org/10.1016/j.bios.2014.08.078.

(41) Wang, X.; Wu, Q.; Shan, Z.; Huang, Q. BSA-Stabilized Au Clusters as Peroxidase Mimetics for Use in Xanthine Detection. Biosens. Bioelectron. 2011, 26, 3614-3619. https://doi.org/10.1016/j.bios.2011.02.014.

(42) Feng, J.; Huang, P.; Wu, F. Gold-Platinum Bimetallic Nanoclusters with Enhanced Peroxidase-like Activity and Their Integrated Agarose Hydrogel-Based Sensing Platform for the Colorimetric Analysis of Glucose Levels in Serum. Analyst 2017, 142, 4106-4115.

(43) Gao, L.; Zhuang, J.; Nie, L.; Zhang, J.; Zhang, Y.; Gu, N.; Wang, T.; Feng, J.; Yang, D.; Perrett, S.; Yan, X. Intrinsic Peroxidase-like Activity of Ferromagnetic Nanoparticles. Nat. Nanotechnol. 2007, 2 (9), 577-583. https://doi.org/10.1038/nnano.2007.260.

(44) Xian-ming, Q.; Liu, Z.; Cai, S.; Zhao, Y.; Wu, D. Electrochemical Aptasensor for the Detection of Vascular Endothelial Growth Factor ( VEGF ) Based on DNA-Templated Ag / Pt Bimetallic Nanoclusters. Chin. Chem. Lett. 2016, No. 3677, 1-7. https://doi.org/10.1016/j.cclet.2016.04.014. 
Graphical Abstract

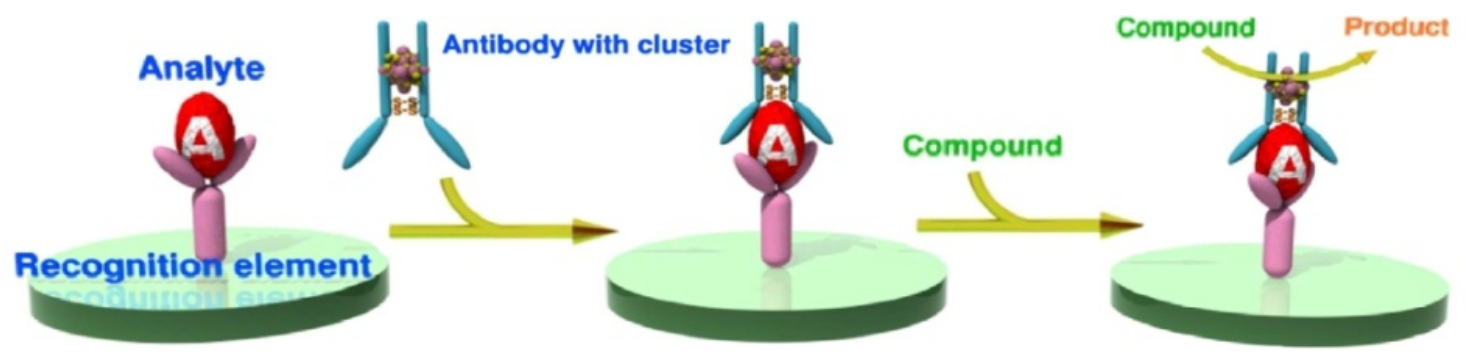

31 